)

0

101,50 LA-5010

MASTER

Multiple-Pass Laser Heating of a Magnetically Confined Plasma 
This report was prepared as an account of work sponsored by the United States Government. Neither the United States nor the United States Atomic Energy Commission, nor any of their employees, ncr any of their contractors, subcontractors, or their employees, makes any warranty, express or implied, or assumes any legal liability or responsibility for the accuracy, com. pleteness or usefulness of any information, apperatus, product or process disclosed, or represents that its use would not infringe privately owned rights.

Printed in the United States of America. Available from National Technical Information Service

U. S. Department of Commerce 5285 Port Royal Road Springfield, Virginia 22151

Price: Printed Copy \$3.00; Microfiche $\$ 0.95$ 


\section{Multiple-Pass Laser Heating of a Magnetically Confined Plasma}

by

Stanley Humphries, Jr.

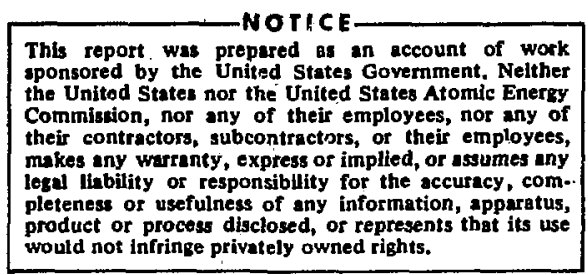

This work was supported by the Presidential Internships in Science and Engineering, funded by the U. S. Labor Department from its Technology Mobilization and Reemployment Program. 
MULIPLE-PASS IASER HEATING OF A MAGETICALLY CONFINED PLASMA

by

Stanley Humphries, Jr.

ABSTRACT

\begin{abstract}
If the plasma to be heated is placed Inside the optical cavity of a laser, a multiple-pass system allowing on the order of 100 passes thi ough the plasma can result. Plasma conditions necessary to assure a stable cavity are derived. An experiment 18 described in which it 18 expected to aignificantly raige the temperature of plasma produced in a z-pinch entirely by Inverse Bremsstrahlung. It may be posstble to use the plasma to $Q-8 w 1$ tch the laser to make more effictent use of the aval lable energy.
\end{abstract}

\section{INTRODUCTICN}

In this report a multiple-pass arrangement 18 described for heating a magnetically confined plasma. FIgure 1 shows that the plasme lo located within the optical cavity of the leser and allows perhaps 100 passes through the plasma. We w1ll show that stable cavities can result even with very long plasma columns. Use of the closed cavity sys tem to Investigate laser-plasma Interactions in current laboratory plasmas will be emphasized. Numerical examples will be based on an experiment curxently in preparation. Using the plasme pro. duced by a stabilized $z$-pinch should allow posstble absorption of almst the entire energy of a $20 \mathrm{~J}$ pulsed $\mathrm{CO}_{2}$ laser. Interpretation of results will be facflitated by the highly uniform radiation dengity throughout the length of the plasma column.

Dawson et al. ${ }^{1}$ show that heating a magnetically confined plagma is a concelvable procedure for obtaining fusion conditions. The long absorption length for the radiation coupled with end losses from the proposed 6 -pinch configuration necessitates on the order of a 1-km-long system. The resulting large plasma volume would require a laser with an output power of 1 to $10 \mathrm{~mJ}$. If the end loss problem cisn be alleviated, the multiple-pass arrangement described may reduce the problem of long absorption lengths.

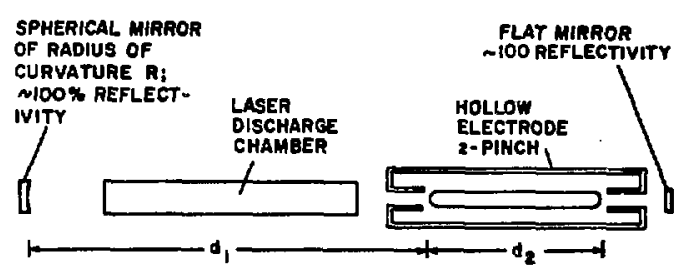

Fig. 1. Experimental arrangement for multiple-pass laser plasma heating.

\section{STÁBILITY OF THE IASER CAVITY}

A hemispherical cavity has been chosen in

Fig. 1 to minimize the width of the laser mode within the plasma and to maximfe the width in the laser discharge chamber. A primary question is whether or not this configuration can be stable. A simple model using geometric optica will be used to investigate stability criteria. Figure 2 shows an infinite optical cavity equivalent to that of $\mathrm{Fig}$. 1 with approprlate ray transfer matrices ${ }^{2}$ entored under the various elements. The plasma is assimed to have a length $d_{2}$ with sharp axial boundaries. Because it is expected that $\omega_{p}^{a} \ll \omega^{2}$, the average index of refraction closely equals 1 , and reflection at the boundartes 18 negligible. Over the crosssectional area filled by the laser mode, radial electron density variations are approximated by a parabolic dependence ouch as

$$
n_{e}(r)=n_{e o}\left(1 \pm \alpha r^{3}\right)
$$


In the limit that $\omega_{p}^{2} \ll w^{2}$, the index of refraction varies radially as

$$
n(r) \approx 1 \pm n_{2} r^{2} \text { where } n_{2}=\alpha \frac{\omega_{p}^{2}}{\omega^{2}}
$$

For the minus $8 \mathrm{ign}$ (corresponding to a hollow density distribution), the ray transfer matrix for the plasma is that given In Fig. 2. For the plus sign the trigononetric functions are replaced by hyperbolls functions. The total ray transfer matrix for one pass through the system is computed by multiplication of the individual matrices. The system is stable if the magnitude of the trace of the total matrix is less than 2. This Implies that

$$
-\left(\frac{1}{R \sqrt{n_{2}}}+\sqrt{n_{2} d_{1}\left(1-\frac{2 d_{1}}{R}\right) \cos 2 \sqrt{n_{2}} d_{2}}\right.
$$

Fig. 2. Optical equivalent of $\mathrm{F} I g .1$.

for the hollow plasma density distribution. Here $R$ Is the radius of curvature of the spherical mirror, and $d_{1}$ is the distance from this mirror to the plasma boundary.

When the product $2 \sqrt{\pi_{2}} d_{2}$ is of the order of 1 or larger, refraction effects in the plasma are significant. For almcst all practical cases density distributions decreasing from the center are unstable. Thus only hollow distributions, such as are expected to occur in the Initial implosion of a pinch, w111 be of use.

For a hollow profile the two terms in Eq. (3) w111 be considered separately. When $2 \sqrt{n_{2}} d=0, \pi$, $2 \pi, \ldots$ the condition for stability becomes -1 < $\left(1-2 d_{1} / R\right)<+1$, equivalent to the result obtained for a normal laser if the length occupied by the plasma is neglected. This occurs because the plasma ray transfer matrix becomes unity in this limit, and all rays leave the plasma with the same displacement and angle with which they entered. When $2 \sqrt{\pi_{2}} d_{2}=\pi / 2,3 x / 2, \ldots$, the stability condition 18

$$
\left|\frac{1}{R \sqrt{n_{2}}}+d_{1} \sqrt{n_{2}}\left(1-d_{1} / R\right)\right|<1 .
$$

Depending on choices of $d_{1}$ and $R$ relative to $n_{2}$, the cavity may be efther stable or unstable in this 1 init.

During the compression phase of the plasma, the average electron density Increases, and the redial extent of the plasma decreases. The corresponding Increase in $\mathrm{n}_{2}$ may cause the product $2 \sqrt{n_{2}} d_{2}$ to pass through one or more of the 1 imfts mentioned above. This ralses the possibility of switching the laser using the plasma by a suirable choice of parameters so that the 1 imit represented by Eq. (4) is unstable. In this way it may be possible to double pulse the laser to use more efficientiy the initial population Inversion. It should be remembered, however, that, because of their large gain (over 50\%), $\mathrm{CO}_{2}$ lasers can lase even with an unstable cavity. More detailed calculations are necessary to determine if losses can be made high enough in the unstable limit to prevent las ing.

The following example illugtrates that, with parameters typical of the experiment in preparation, It is possible to achieve both stable and unstable 1imits. The quantity $n_{e o} 18$ chosen to equal $5 \times 10^{16} \mathrm{~cm}^{-3}$, giving $w_{p}=1.3 \times 10^{13} \mathrm{sec}^{-1} \mathrm{com}-$ pared to the laser frequency (for $10.6 \mu$ radiation of $\omega=1.8 \times 10^{14}$ ). We assume that there is a $20 \%$ rise in density $1 \mathrm{~cm}$ from the axis, implying that $\mathrm{n}_{2}=10^{-3} ; \mathrm{d}_{2}=30 \mathrm{~cm}$ so that $2 \sqrt{\mathrm{n}_{2}} \mathrm{~d}_{2}=1.9$, we11 into the refraction dominated case. Hopefully it will be possible to extend $2 \sqrt{n_{2}} d_{2}$ to 3.14 .

Where $2 \sqrt{n_{2}} d_{2}=1.57$, the ratio of $d_{1}$ and $R$ can be adfugted, $d_{1}=r / \alpha$. Bquation (4) becomes

$$
R^{2} \sqrt{n_{2}}(1-1 / \alpha)-R+\frac{1}{\sqrt{n_{2}}}=0
$$

A real solution occurs if $1<\alpha<1.33$. Th1s constrains $R$ such that $1 / \sqrt{n_{2}}<R<2 / \sqrt{n_{2}}$. In the 
example used, $R$ must be less than 63 , and hence $d_{1}$ is less than $47 \mathrm{~cm}$. The discharge chamber used has a length of about $1.5 \mathrm{~m}$ so that in this limit the cavity should be anstable.

The paraxial ray approximation has been used in the above analysis. It is also necessary to show that the laser mode pattern occuples a $8 \mathrm{~ms} 11$ crosssectional area compared to the plasma. In the following, we assume the $T E I_{00}$ mode, $2 \sqrt{\pi_{2}} d_{2}=\pi, d_{2}=$ $30 \mathrm{~cm}$, and $2 \mathrm{~d}_{1}=\mathrm{R}$. The beam width at the entrance to the plasma $18 \mathrm{w}_{0}-\lambda_{\mathrm{d}_{1}} / \pi$, and the beam width $a$ : the spherical mirror is $w_{s}=\sqrt{2} w_{0}$. Rays entering with displacement $x_{1}$ and zero entrance angle oscillate about the axis with maximum displacement $x_{1}$. The maximum displacement is of the order of $w_{0}$ that, for $d_{1}=2 \mathrm{~m}$, equals $2.6 \mathrm{~mm}$. Rays entering with zero displacement and angle $x_{1}$ ' resch a maximum displacement $x_{2}=x_{1}^{\prime} / / n_{2}$. The maximum angle $1_{s}$ approximately $w_{s} / d_{1}$ so that the maximum displacement is about $0.3 \mathrm{~mm}$. Thus the modis would be expected to have a widh of less than $3 \mathrm{~mm}$, which should be small conpared to the radius of the plasma.

There is a case of light propagation through the plasma that has an exact silucion and is of particular interest in the heating of a long plasma column. If a Gaussian beam, propagating through a plasma whose index of refraction is given by Eq. (2), has an infinite radius of curvature and a beam width given by

$$
w=\left(\frac{\lambda}{\pi}\right)^{\frac{1}{2}}\left(\frac{1}{n_{2}}\right)^{\frac{1}{4}},
$$

then the beam w111 propagate indefinitely thout a shange in 1 ts parameters. ${ }^{3}$ If $\mathrm{n}_{2}$ is known, additional optics can be introduced into the discharge chamber to match modes at the plasme boundary. Because $w$ is an Insensitive function of $n_{2}$, the match need not be exact. of particular interest is the fact that $w 18$ sma 11 , even for very 8 mall $n_{2}$. For Instance, with $\lambda=10.6 \mu$ and $n_{2}=10^{-3}$, w equals 1 m. Thus the beam can be made thin compared to the plasma column even for long plasmas. The resulting strong localized heating coupled with the fact that only a small $n_{2}$ is necessary makes $1 t$ more probable that the radiation can maintain a hollow density profile along the axis of the plasma.

A long plasma would thus act as a dielectric waveguide. Lasers incorporating such waveguides within their optical cavities have been operated success fully."

\section{PIASMA REQUIREMENTS}

For the experiment to be meatingful, the plasma energy should be changed significantly by the laser radiation. Also if the absorbed energy is comparable to or greater than the initial energy of the plasma, therc is a chance that the heating, atrongly localized at the center, will help to maintain the hollow density profile. The nature of the plasma is determined mainly by the available laser energy.

To get a rough estimate of the desired parameters, we assume that the plasma has some initial spatially averaged density and electron temperature, $\mathrm{n}_{e o}$ and $\mathrm{kT}_{e}$. The inverse Bremsstrahlung absorption length will be calculated from these quantities. The plasma has length $d_{2}$ and cross-sectional area A. Assuming $T_{i} \sim T_{e}$, the initial energy is

$$
E_{p}=2 A_{2} n_{e o} \mathrm{kT}_{e}
$$

The inverse Bremsstrahlung absorption length is given by

$$
I=\frac{5 \times 10^{27}\left(\mathrm{kT}_{\mathrm{e}}\right) \not \beta}{n_{\mathrm{eo}}{ }^{2} 2 \lambda^{2}}
$$

with $\mathrm{n}_{\mathrm{eo}}$ in $\mathrm{cm}^{-3}, \mathrm{kT}_{\mathrm{e}}$ in $\mathrm{eV}$, and $\lambda$ in $\mathrm{cm}$. For $d_{f} \ll I$, the fractional absorption in one pase through the plasma is $\mathrm{d}_{2} / \mathrm{L}$. If $\mathrm{T}$ represents the fractional losses due to other causes (1.e., mirror 10s8), then the fraction of absorbed energy lost per pass through the system is

$$
f-\frac{d_{2} / L}{d_{2} / L+T} \text {. }
$$

Al1 energy $1 \mathrm{~s}$ eventually lost; therefore the cotal energy absorbed by the plasme is $\mathrm{fE}_{\ell}, \mathrm{E}_{\ell}$ being total energy of the laser pulse. The criterion for significant plasma heating - that the aboorbed energy be equal to or greater than the inftial plasma energy - can be written

$$
\mathrm{FE}_{\ell}>\mathrm{E}_{\mathrm{P}}
$$


Figure $3 a$ showe a plot of permissible values of $\mathrm{n}_{\mathrm{eo}}$ vs $\mathrm{kT}$ for the following parameters: $\omega=1.8 \times 10^{14}$ $\sec ^{-1} ; A=3 \mathrm{~cm}^{2} ; d_{2}=30 \mathrm{~cm} ;$ and $E=20 \mathrm{~J}$. Because mIrrors having $R=0.995$ are currently avallable, $T$ is chosen equal to 0.005 . Figure $3 \mathrm{~b}$ shows a plot of total energy absorbed for $\mathrm{n}_{e o}$ and $\mathrm{kT}_{\mathrm{e}}$ on the limiting line of $\mathrm{Fig}$. 3a. The best region is that between $n_{e o}=10^{16}-10^{17} \mathrm{~cm}^{-3}$. Here a large fraction of the laser energy is absorbed, and the highest Initial plasma temperatures are permissible.

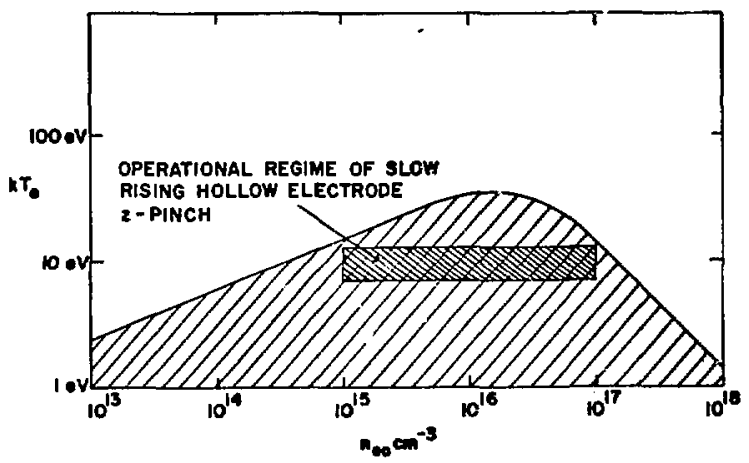

Fig. 3a. Permissible plasma parameters allowing absorbed energy to be equal to or greater than the inttial plasma energy. $(E=20$ $\left.J, T=0,005, A=3 \mathrm{~cm}^{2}, d_{2}=30 \mathrm{~cm}\right)$

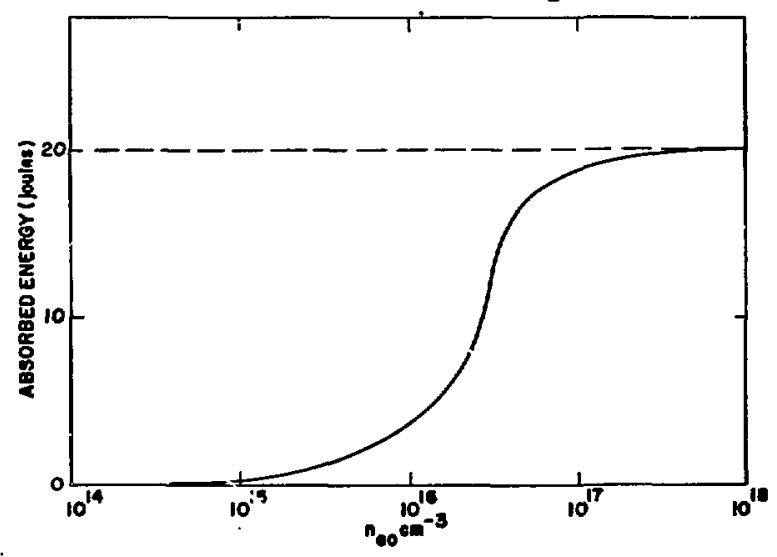

Fig. 3b. Absorbed energy for $n$ and $k T$ taken on the limiting curve of ${ }^{e}{ }_{1 g} \cdot 3 a \cdot{ }^{e} E_{\ell}=20 \mathrm{~J}$.

\section{EXPER TYENTS}

A transverse discharge, $\mathrm{CO}_{2}-\mathrm{N}_{2}$, laser with an expected $\mathrm{E}_{\ell}$ of $20 \mathrm{~J} \mathrm{Is}$ under construction. A $30-\mathrm{cm}$ $z$-pinch with parameters expected in the range shown in Fig. 3a is also being developed. Because this device is being constructed to test diagnostica measuring $\mathrm{n}_{e}(r), k T_{e}$, and $k T_{1}$, it will be convenient to incorporate the experiment discussed. The expected range of parameters of the $z$-pinch is plotted in Fig. 3a.

Faraday rotation of the laser radiation passing through the plasma column is caused by axial flelds. In the z-pinch an axial stabilizing field in excess of. $1 \mathrm{kG}$ will be necessary. In one double pass through the plasma, the plane of polarization of the radiation will be rotated an angle

$$
\theta=\left(\frac{w_{p}}{\omega}\right)^{3} \frac{w_{b} d_{2}}{c} \text { where } \omega_{b}=\frac{e B_{z}}{m_{e}} \text {. }
$$

with $B_{z}=1000 \mathrm{~g}, n_{e o}=5 \times 10^{16}$, and $\theta=1.8 \times 10^{-2}$. The Brewster windows, which totally transmit only one pclarization, will cause excessive losses because the radietion must make many passes through the system. We plan to use dielectric coated Itran windows to separate the discharge chamber from the z-pinch.

If lasing can be achleved, the closed cavity system seems attractive for heating experiments on current laboratory plasmas and also may prove useful in larger plasma devices. Q-switching risetimes of the order of $100 \mathrm{nsec}$ have been achleved In a relatively long $\mathrm{CO}_{2}$ laser ${ }^{6}$ so that plasma switching of the laser should be possible if losses can be made high enough in the unstable limits. This will ensure that the laser fires only when the radiation can be contained by the plasma.

\section{ACKNOWLEDGMENTS}

I would like to thank $W$. Condtt of LLL and P. Forman, R. Morse, and R. Mjolsness of LASL for many helpful suggestions.

\section{REFERENCES}

1. J. W. Dawson et al., "Controlled Eusion Using Long Wavelength Laser Heating with Magnetic Confinement," Paper No. CN-28/D-1.3, IAEA Conference, Madison, Wisconsin June 1971.

2. H. Kogeinik and T. L1, "Laser Beams and Resonators," App1. Opt. $\underline{5}$, No. 10, 1550 (1966).

3. A Yariv, Introduction to Optical Electronica (Holt, Rinehart and Winston, Inc., New York, 1971) p.37.

4. P. W. Smith, "A Waveguide Gas Laser," Appl. Phys. Lett. 19, No. 5, 132 (1971).

5. P. Porman, Los Alamos Scientific Laboratory, private communication, March 1972. 
6. T. J. Bridges and P. R. Cheo, "Spontaneous Self-Pulsing and Cavity Dumping in a $\mathrm{CO}_{2}$ Laser with E lectro-optic Q-8witching," App1. "Phys.

Lett. 14, No. 9, 262, (1969) 